\title{
VARIATIONS OF THE SUPERFICIAL PALMAR ARCH: A CLINICO- ANATOMICAL CONSIDERATION
}

\author{
Pooja Dawani ${ }^{1}$, Anita Mahajan ${ }^{* 2}$, Sabita Mishra ${ }^{3}$, Neelam Vasudeva ${ }^{4}$. \\ ${ }^{1}$ Senior Resident of Anatomy, Maulana Azad Medical College, New Delhi, India. \\ *2 Director Professor of Anatomy, Maulana Azad Medical College, New Delhi, India. \\ ${ }^{3}$ Director Professor and Head of Anatomy, Maulana Azad Medical College, New Delhi, India. \\ ${ }^{4}$ Ex. Director Professor of Anatomy, Maulana Azad Medical College, New Delhi, India.
}

\section{ABSTRACT}

\begin{abstract}
Introduction: The superficial palmar arch plays a vital role in the blood supply to the hand. The knowledge of variations in the pattern of superficial palmar arch and its branches, and caliber of these arteries, has become more important for surgeons in reconstructive hand surgeries and radial artery harvesting for myocardial revascularization. The aim of this study was to observe the variations and measure the diameter of contributing arteries of the arches.
\end{abstract}

Materials and methods: In the present study, thirty hands from formalin fixed adult human cadavers were dissected. Normal pattern as well as variations in the arteries contributing to the arch, completeness of arch, and the branching pattern were observed and noted. With the help of a digital caliper, the diameters of ulnar and radial arteries and median artery were taken at the level of the wrist and statistical analysis of the results was done.

Results: It was observed that in $96.7 \%$ cases, the superficial palmar arch was complete where there was anastomosis between the arteries forming the arch. In 3.3\% cases, the arch was incomplete. The complete arch was divided into radioulnar (36.7\%), ulnar (56.7\%) and medianoulnar (3.3\%) types. The diameter of superficial palmar branch of radial artery was found to be significantly less than the diameter of ulnar artery. $(p=0.003)$.

Conclusion: The data obtained on variations and morphometry of superficial palmar arches will certainly be useful in planning and choosing appropriate and safe surgical procedures to prevent inadvertent outcome.

KEY WORDS: Palmar arch, blood supply, Hand, radial artery, myocardial revascularization.

Corresponding Author: Dr Anita Mahajan, Director Professor of Anatomy, Maulana Azad Medical College, New Delhi, India. E-Mail: anitamahajan24@gmail.com

Access this Article online

Quick Response code

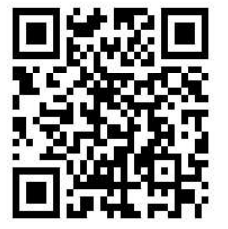

DOI: 10.16965/ijar.2020.231
Journal Information

International Journal of Anatomy and Research ISSN (E) 2321-4287 | ISSN (P) 2321-8967 https://www.ijmhr.org/ijar.htm DOI-Prefix: https://dx.doi.org/10.16965/ijar

\section{Article Information}

Received: 04 Oct 2020

Peer Review: 04 Oct 2020

Revised: None
Accepted: 13 Nov 2020

Published (O): 05 Dec 2020

Published (P): 05 Dec 2020

\section{INTRODUCTION}

Radial and ulnar arteries anastomose at various sites at the wrist and in the hand to form palmar and dorsal carpal arches, superficial and deep palmar arches, anastomosis between palmar metacarpal branches of deep palmar arch and common palmar digital branches of superficial palmar arch. The superficial palmar arch plays a vital role in the blood supply to the hand.

With recent advances in microsurgical techniques in reconstructive hand surgery, the knowledge of variations in the pattern of arterial arches and their branches, and 
caliber of these arteries has become more important for surgeons. Radial artery harvesting for myocardial revascularization is another procedure that is popular now a days. It is done in addition, or as alternative to venous or other arterial graft. This procedure carries potential risks, as confirmed by a report of ischemia of hand after radial artery removal [1]. This complication occurs due to absence of collateral circulation from the ulnar artery. The risk is also involved in other procedures like radial artery forearm flap, radial artery cannulation, etc. These procedures can be performed successfully only if there is a well-established collateral circulation between the arteries supplying the hand. Non-invasive methods such as Allen's test and Doppler ultrasonography can be done to assess the hand circulation, but they are not reliable in all cases. Thus a background of detailed anatomical knowledge derived from gross dissection is needed before performing any procedure on the upper extremity.

Anatomical studies on morphometry of arteries provides the knowledge of diameter of arteries supplying the hand which can help in improving microsurgical techniques in reconstructive hand surgeries and in proposing new surgical techniques.

The objective of this study was to observe the variations encountered in the superficial palmar arches and note their prevalence, and to measure the diameter of contributing arteries of the arches. This will certainly be helpful in planning and improving microsurgical techniques like reconstructive hand surgery and radial artery harvesting, to achieve the best results and also proposing newer and safer surgical procedures.

\section{MATERIALS AND METHODS}

The present study was conducted in the Department of Anatomy, Maulana Azad Medical College, New Delhi. Thirty hands from formalin fixed adult human cadavers were dissected. Normal pattern as well as variations in the arteries contributing to the arch, completeness of arch, and the branching pattern were observed and noted. With the help of a digital caliper, the diameters of ulnar and radial arteries and median artery (where present) were taken at the level of the wrist.

Statistical analysis: Mean and standard deviation of the diameter of arteries were calculated. Student's t test was performed to compare the diameters of arteries in the same hand, and also between right and left hands. $\mathrm{P}<0.05$ was considered significant.

\section{RESULTS}

The superficial palmar arch is divided into two groups on the basis of completeness of the arch:

Group I: Complete arch: An anastomosis takes place between the arteries that form the arch. In the absence of a contribution from the radial or the median artery, the ulnar artery is considered to form a complete arch if it supplies all fingers as well as the thumb. In present study, complete SPA was observed in $96.7 \%$ cases.

Group II: Incomplete arch (Fig 1): The arch is said to be incomplete, when the contributing arteries to the superficial arch do not anastomose, or when the ulnar artery fails to reach the thumb and the index finger.

This arch was seen in one out of 30 hands (3.3\%).

In this case, superficial palmar branch of the radial artery supplied the thumb and radial side of index finger. The ulnar artery gave proper palmar digital branch to the ulnar side of little finger, and common palmar digital branches to the adjacent sides of the little, ring and middle fingers. The perforating artery from the palmar metacarpal artery in the second web space supplied the adjacent sides of the index and middle fingers.

The complete arch was divided into subtypes based on the contribution of the arteries forming the arch:

Type A: Radioulnar type: This type of superficial palmar arch was formed by the superficial palmar branch of the radial artery and the terminal branch of the ulnar artery. It was found in 11 hands (36.7\%) This type was subdivided into the following two subtypes: 
Subtype 1. Predominant ulnar artery type: This consists of a large terminal branch of the ulnar artery and the thin superficial palmar branch of the radial artery. It was noted in 9 hands (30.0\%). (Fig.2)

Subtype 2. Equal type: The contribution of the ulnar artery and superficial palmar branch of the radial artery appeared almost equal. It was seen in 2 hands (6.7\%).

Type B: Ulnar type: This type consists entirely of the ulnar artery, which continues as far as the index finger and thumb without any connection with other arteries. This type was seen in 17 hands (56.7\%). (Fig.3)

Type C: Medianoulnar type: This type is formed by the terminal portion of the ulnar artery and the palmar type of median artery. It was seen in one hand (3.3\%). (Fig.4)

The branching pattern of the arch was classified on the basis of the number of direct branches from each arch:

Pattern 1: The four common palmar digital arteries to the corresponding interdigital webs and the proper palmar digital artery to the little finger, originated directly from the superficial palmar arch. It was seen in 26 hands (86.7\%).

Pattern 2: The fourth common palmar digital artery and the proper palmar digital artery to the little finger originated from a common trunk, which in turn, originated directly from the superficial palmar arch. The first, second and third common palmar digital arteries originated directly from the superficial palmar arch. This pattern was seen in 3 hands (10\%).

Pattern 3: The fourth common palmar digital artery and the proper palmar digital artery to the little finger originated from a common trunk. The second and third common palmar digital arteries also originated from a common trunk. (Fig. 5)

The diameters of forming arteries of supe rficial palmar arch are shown in table 1 . The diameter of superficial palmar branch of radial artery was found to be significantly less than the diameter of ulnar artery in the same hand. $(p=0.003)$. There was no significant difference in the diameter of arteries in the right and left hands.
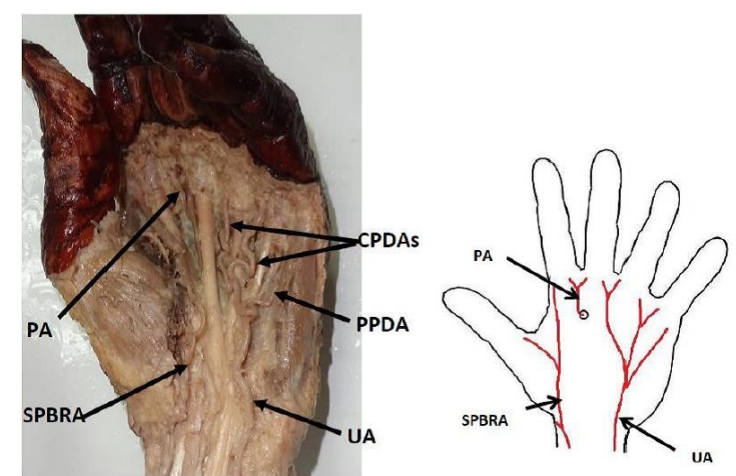

Fig. 1: Incomplete superficial palmar arch (image and schematic diagram), UA- Ulnar artery, SPBRA-superficial palmar branch of radial artery CPDA- common palmar digital artery, PPDA-proper palmar digital artery, PA-perforating artery from deep palmar arch.
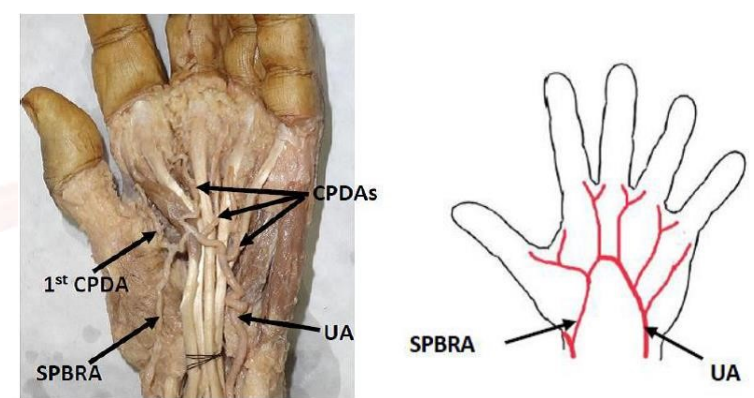

Fig. 2: Radioulnar type of complete superficial palmar arch (image and schematic diagram). UA- Ulnar artery, SPBRA-superficial palmar branch of radial artery CPDA- common palmar digital artery.
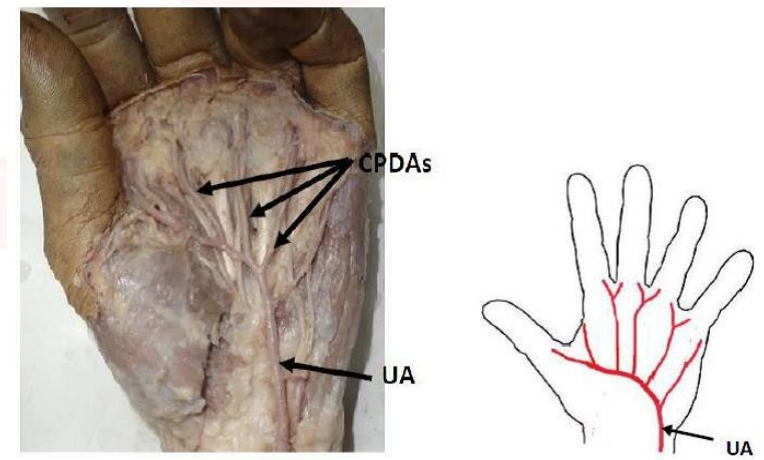

Fig. 3: Ulnar type of complete superficial palmar arch. (Image and schematic diagram) UA- Ulnar artery, CPDAcommon palmar digital artery.
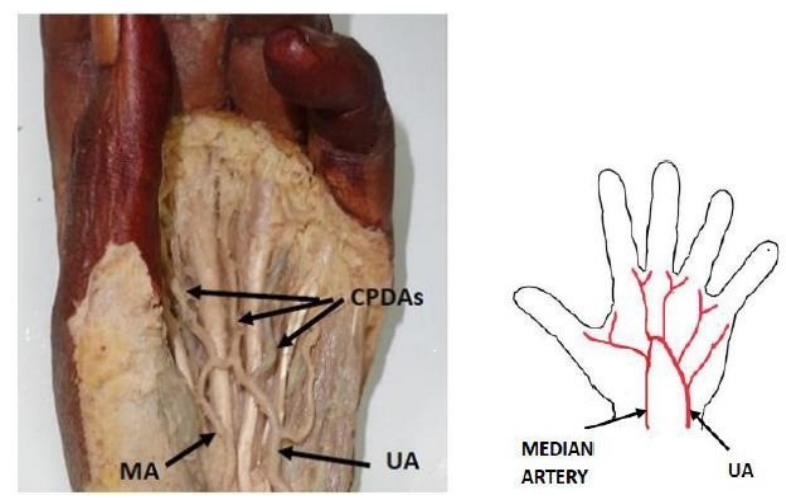

Fig. 4: Medianoulnar type of complete superficial palmar arch. (Image and schematic diagram) UA- Ulnar artery, MA- Median artery, CPDA- common palmar digital artery. 

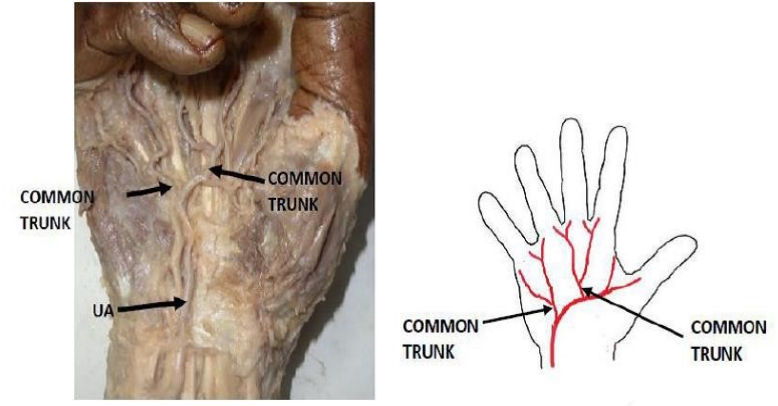

Fig. 5: Branching pattern of superficial palmar arch showing two common trunks (image and schematic diagram). UA- Ulnar artery.

Table 1: Showing the diameters of forming arteries of superficial palmar arch.

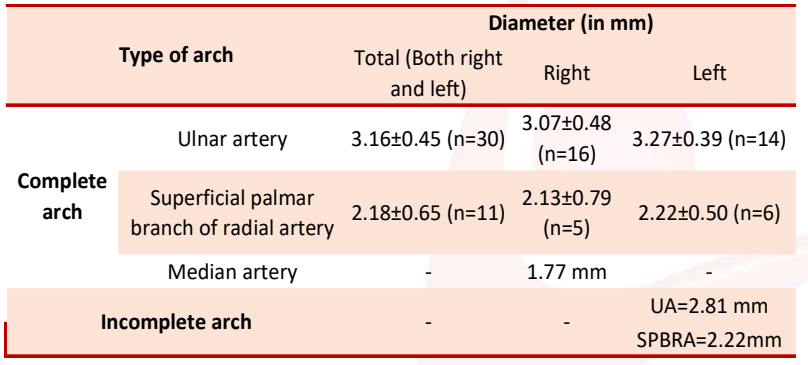

Table 2: Comparing the findings of present study with previous studies.

\begin{tabular}{ccccc} 
Parameter Studied & Fazan [21] & Bilge [10] & Gellman [11] & Present Study \\
\hline \multirow{2}{*}{ UA (diameter in mm) } & $\mathrm{R}-2.5$ & $\mathrm{R}-3.57$ & \multirow{2}{*}{2.55} & $\mathrm{R}-3.07$ \\
& $\mathrm{~L}-2.6$ & $\mathrm{~L}-3.59$ & & $\mathrm{~L}-3.27$ \\
\multirow{2}{*}{ SPBRA (diameter in mm) } & $\mathrm{R}-1.7$ & $\mathrm{R}-1.7$ & \multirow{2}{*}{1.6} & $\mathrm{R}-2.13$ \\
& $\mathrm{~L}-1.4$ & $\mathrm{~L}-1.65$ & & $\mathrm{~L}-2.22$ \\
\hline
\end{tabular}

\section{DISCUSSION}

The occurrence of variations encountered in this study can be explained by understanding the process of development of arterial system of upper limb. The anomalies of upper limb arteries occur due to inhibition of the development of the vessels at different stages of the embryonic period [2].

The limb buds appear from the ventrolateral body wall during the fourth week in embryo [3], and a primitive capillary plexus is formed in the mesenchyme. This establishes connection with the branches of intersegmental arteries arising from the aorta. The subclavianaxillary artery is the only arterial stem to supply the upper limb [4]. The primitive vascular pattern has a primary axial artery as the brachial artery in the arm and interosseous artery in the forearm. The median artery is branch from the brachial artery, which makes connection with the vessels of hand. The ulnar artery arising from the brachial artery unites distally with the median artery to form the arch pattern [2]. Then the radial artery arises from the brachial artery and finally takes over the vessels of the hand.

Manners Smith attempted to describe the variations of hand arteries phylogenetically. He made a comparative study of arteries of the hand in primates and concluded that many of the variation observed in man represent a retention or reappearance of primitive patterns [5].

O'Sullivan and Mitchell associated the absence of the palmaris longus tendon with the variant patterns of SPA. However, in the present study, this tendon was present in all cases [6]. In the present study, complete SPA was found in $96.7 \%$ cases and incomplete SPA in $3.3 \%$ cases. The results are similar to that of Ikeda and Loukas who found complete arches in $96.4 \%$ and $90.0 \%$ respectively. ${ }^{[7,8]}$ Ikeda studied SPA using both arteriography and dissection, which may determine the higher percentage found in their study [7]. In the present study, the findings can be attributed to the small sample size.

Standard anatomical textbooks describe that the superficial palmar arch is formed by anastomosis of superficial palmar branch of radial artery and the continuation of ulnar artery. In contrast, we found this classical arch in $36.7 \%$ cases only, agreeing with findings of other authors [9-11].

In the present study, $56.7 \%$ of SPAs were formed by ulnar artery only. This finding disagrees with other studies. Coleman and Anson found ulnar arches in $37.0 \%$ cases [9]. Other studies also report percentages between $30 \%$ and $40 \%[8,9,11]$.

The median artery represents the arterial axis of the forearm during early embryonic life. It normally regresses in the second embryonic month, but may persist in 2 patterns, as the palmar type that reaches palm and forms superficial palmar arch, or as the antebrachial type that ends before reaching the wrist joint [12]. The complete arch formed by the ulnar artery and median artery was observed in 3.3\% cases in the present study, similar to the findings of other authors $[9,10,13]$. Loukas found 
the medianoulnar arch in $15 \%$ cases, higher in incidence than the present study [8].

Other arteries that can complete the superficial palmar arch on the radial side include the princeps pollicis and the radialis indicis arteries, as reported in previous studies [14-16]. Jelicic observed anastomosis of ulnar artery with the first dorsal metacarpal arteries in 20 $\%$ cases [17]. These variants of the superficial palmar arch were not reported in the present study.

The incomplete superficial palmar arch was observed in only $3.3 \%$ hands in the present study. It showed an unusual pattern, where the superficial palmar branch of radial artery supplied thumb and radial side of index finger, and the ulnar artery supplied branches to third and fourth web spaces. The second web space received contribution from the perforating branch of palmar metacarpal artery. Bataineh has reported a similar case previously [18].

Conventionally, the superficial palmar arch is said to give three common palmar digital arteries to the second, third and fourth interdigital webs. Tandler observed an artery in the first interspace, which supplied branches to the thumb and index finger and called it the first common palmar digital artery [19]. Coleman and Anson also found 4 CPDAs in $77.3 \%$ of cases [9]. In the present study, the superficial palmar arch gave 4 CPDAs; and a PPDA to the ulnar side of little finger. The classification was based on whether the branches originated directly or indirectly from the arch. The fourth CPDA and PPDA arose from common trunk in 3 cases, and in 1 case, the second and third CPDA also arose from common trunk. Onderglu reported a case in which first and second CPDAs arose from a common trunk [20].

The average UA diameter measured by Bilge et al is larger than that found in the present study [10]. On the other hand, Fazan recorded smaller values [21]. This difference can be attributed to the ethnic and/or environmental variations. The superficial palmar branch of radial artery showed larger diameter values in the present study, suggesting its significant role in formation of SPA and blood supply to fingers. Gellman et al recorded smaller values as they measured the intraluminal diameters of arteries using resin injection and cast formation technique [11]. (Table 2)

If the superficial palmar arch is complete, it appears safe to sacrifice the radial artery in arterial interventions like radial artery cannulation, radial artery forearm flap, and harvesting of radial artery to serve as bypass conduit in coronary artery disease. However, the decision to remove radial artery depends on factors like the type of complete SPA, the caliber of anastomosing vessels and their functionality, concomitant ulnar artery pathology, etc. The median artery is given by ulnar artery in the forearm, thus in cases of bleeding from hand, ulnar artery ligation close to its origin, may compromise the blood flow to the fingers. Also, if the radial artery has been removed already for bypass surgery, the blood supply to the hand will be completely jeopardized on ligation of ulnar artery. In the equal subtype of radioulnar complete arch, the removal of radial artery will have higher chances of ischemia of fingers because the ulnar artery alone will not be sufficient to supply all fingers. The functionality of anastomoses can be assessed by performing angiography prior to surgical interventions.

\section{CONCLUSION}

The superficial palmar arch is found to be complete in most cases. This suggests that, from anatomic perspective it can be safe to sacrifice radial artery in procedures like radial artery harvesting and radial artery flap transfer, but it is always recommended to use Allen's test and Doppler ultrasound to assess hand circulation prior to any surgical procedure on forearm and hand. The variations and morphometric data of the arches will certainly be useful for surgeons while performing reconstructive surgeries on hand and proposing new microsurgical techniques.

\section{ACKNOWLEDGEMENTS}

The authors are grateful to the Department of Anatomy, Maulana Azad Medical College for providing donated cadavers for dissection of 
the superficial palmar arches of hand.

\section{Conflicts of Interests: None}

\section{REFERENCES}

[1]. Nunoo-Mensah J. An unexpected complication after harvesting of the radial artery for coronary artery bypass grafting. Ann Thoracic Surg. 1998;66(3):929-31.

https://doi.org/10.1016/S0003-4975(98)00559-1

[2]. Singer E. Embryological pattern persisting in the arteries of the arm. Anat Rec. 1933;7:263-82. https://doi.org/10.1002/ar.1090550407

[3]. Moore KL, Persaud TVN. Blood supply to the limbs. In: Before We Are Born - The Limbs. 5th ed. Philadelphia: WB Saunders, 1998: 415-6.

[4]. Arey LB. Arteries of the limbs. In: Developmental Anatomy. 1st ed. Philadelphia: WB Saunders, 1966: 358-9.

[5]. Manners-Smith T. The limb arteries of primates. J Anat Physiol. 1910;45:23-64.

[6]. O'Sullivan E, Mitchell B. Association of the absence of palmaris longus tendon with an anomalous superficial palmar arch in the human hand. J Anat.2002;201:405-8. https://doi.org/10.1046/ j.0021-8782.2002.00109.x PMid:12448775 PMCid:PMC1570962

[7]. Ikeda A, Ugawa A, Kazihara Y, Hamanda N. Arterial patterns in the hand based on a three-dimensional analysis of 220 cadaver hands. J Hand Surg. 1988;13:501-9.

https://doi.org/10.1016/S0363-5023(88)80085-6

[8]. Loukas M, Holdman D, Holdman S. Anatomical variations of the superficial and deep palmar arches. Folia Morphol. 2005 May;64(2):78-83.

[9]. Coleman SS, Anson BJ. Arterial patterns in the hand based upon the study of 650 specimens. Surg Gynecol Obstet. 1961;113:409-24.

[10]. Bilge O, Pinar Y, Ozer MA, Gövsa F. A morphometric study on the superficial palmar arch of the hand. Surg Radiol Anat. 2006 Aug;28(4):343-50 https://doi.org/10.1007/s00276-006-0109-9 PMid:16642281

[11]. Gellman H, Botte MJ, Shankwiler J, Gelberman RH. Arterial patterns of the deep and superficial palmar arches. Clin Orthop Relat Res. 2001;383E:41-6. https://doi.org/10.1097/00003086200102000-00007 PMid:11210968
[12]. Rodriguez-Neidenfuhr M, Sanudo JR, Vazquez T, Nearn L, Logan B, Parkin I. Median artery revisited. J Anat. 1999;195:57-63. https://doi.org/10.1017/S0021878299005075 PMid:10473293 PMCid:PMC1467965

[13]. Al-Turk M, Metcalf WK. A study of the superficial palmar arteries using the Doppler Ultrasonic Flowmeter. J Anat. 1984;138(1):27-32.

[14]. Jiji PJ, D'Costa S, Nayak SR, Prabhu LV, Kumar CG, Prakash. A unique variation of superficial palmar arch. International Journal of Anatomical Variations. 2009;2105-7.

[15]. Salgado LSS, Hasan R, Perera AAMMSL, Wijesundara WMRD, Anuradha WK. Variations of the superficial palmar arch in a Sri Lankan perspective. GSTF J Adv Med Res. 2014;1(2):61-7. https://doi.org/10.5176/2345-7201_1.2.25

[16]. Gupta C, Kalthur SG, Nair N, Pai SR. A morphological study of variations of superficial palmar arches of the hand. CHRISMED Journal of Health and Research. 2015 Apr-Jun;2(2):140-4. https://doi.org/10.4103/2348-3334.153259

[17]. Jelicic N, Gajisin S, Zbrodowski A. Arcus palmaris superficialis. Acta Anat. 1988;132(3):187-90.

[18]. Bataineh ZM, MoqattashST. A complex variation in the superficial palmar arch. Folia Morphol;. 2006;65:406-9.

[19]. Tandler J. Zur. Anatomie der arterien der Hand. Anat Hefte. 1897;7:263-82. https://doi.org/10.1007/BF02298035

[20]. Onderglu S, Basar R, Erbil KM, Cumhur M. Complex variation of the superficial palmar arch-Case report. Surg Radiol Anat. 1997;19(2):123-5. https://doi.org/10.1007/s00276-997-0123-6 https://doi.org/10.1007/BF01628138

[21]. Fazan VP, Borges CT, Da Silva JH, Caetano AG, Filho OA. Superficial palmar arch: an arterial diameter study. J Anat. 2004 Apr;204(4):307-11. https://doi.org/10.1111/j.0021-8782.2004.00281.x PMid:15061757 PMCid:PMC1571293

How to cite this article:

Pooja Dawani, Anita Mahajan, Sabita Mishra, Neelam Vasudeva. VARIATIONS OF THE SUPERFICIAL PALMAR ARCH: A CLINICOANATOMICAL CONSIDERATION. Int J Anat Res 2020;8(4.2):78177822. DOI: $10.16965 /$ ijar.2020.231 